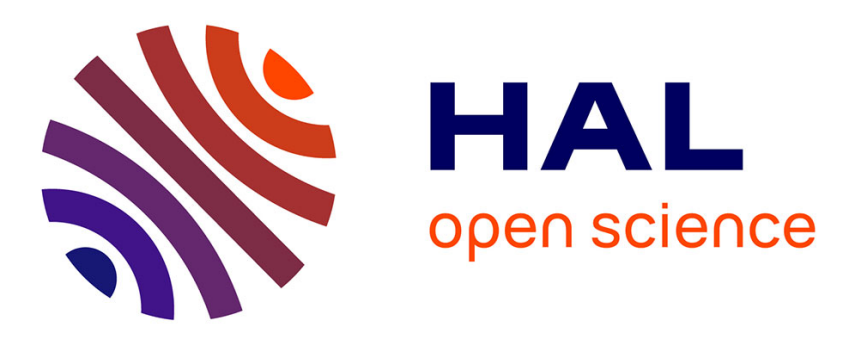

\title{
Heterogeneous nucleation and depletion effect in nanowire growth
}

Fiqiri Hodaj, Olexii Liashenko, Andriy Gusak, Yuriy Lyashenko

\section{To cite this version:}

Fiqiri Hodaj, Olexii Liashenko, Andriy Gusak, Yuriy Lyashenko. Heterogeneous nucleation and depletion effect in nanowire growth. Philosophical Magazine, 2011, 91 (33), pp.4200-4217. 10.1080/14786435.2011.607142 . hal-00728898

\section{HAL Id: hal-00728898 \\ https://hal.science/hal-00728898}

Submitted on 7 Sep 2012

HAL is a multi-disciplinary open access archive for the deposit and dissemination of scientific research documents, whether they are published or not. The documents may come from teaching and research institutions in France or abroad, or from public or private research centers.
L'archive ouverte pluridisciplinaire HAL, est destinée au dépôt et à la diffusion de documents scientifiques de niveau recherche, publiés ou non, émanant des établissements d'enseignement et de recherche français ou étrangers, des laboratoires publics ou privés. 


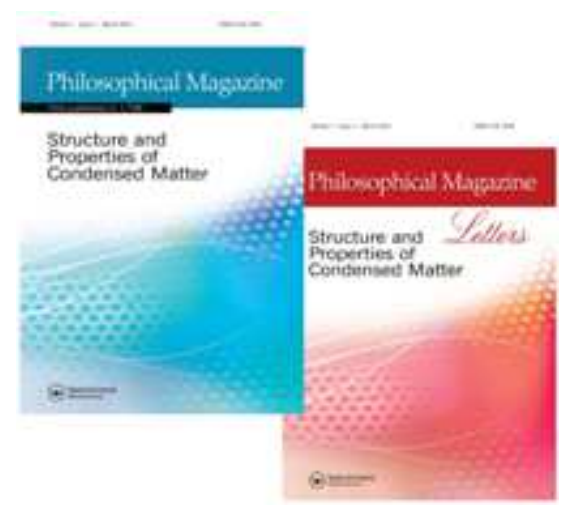

\section{Heterogeneous nucleation and depletion effect in nanowire growth}

\begin{tabular}{|r|l|}
\hline Journal: & Philosophical Magazine \& Philosophical Magazine Letters \\
\hline Manuscript ID: & TPHM-11-May-0172.R1 \\
\hline Journal Selection: & Philosophical Magazine \\
\hline Date Submitted by the & $13-J u l-2011$ \\
\hline Complete List of Authors: & $\begin{array}{l}\text { Hodaj, Fiqiri; Grenoble Institute of Technology, SIMaP, CNRS-UMR } \\
\text { Liashenko, Olexii; Cherkasy National University } \\
\text { Gusak, Andriy; Cherkasy National University } \\
\text { Lyashenko, Yuriy; Cherkasy National University }\end{array}$ \\
\hline Keywords: & nucleation, nanowires, modelling, thermodynamics, growth \\
\hline Keywords (user supplied): & step-flow kinetics \\
\hline \multicolumn{2}{|c}{}
\end{tabular}

\section{SCHOLARONE \\ Manuscripts}




\begin{abstract}
Recent experiments of Ross, Kodambaka et al. proved the possibility of a mononuclear regime with heterogeneous nucleation as well as jerky growth in the VLS process for silicon nanowires. In this work, a theoretical model is presented which incorporates the effects of (i) mononuclear regime with layer by layer growth, (i) heterogeneous nucleation of each new layer at the edge of Au-Si droplet, (iii) drop of supersaturation after each successful nucleation and respective fast layer growth, (iv) time-dependent nucleation barrier during each new waiting period and ( $\mathrm{v}$ ) correlation between subsequent waiting periods (nonMarkovian sequence of waiting periods).
\end{abstract}

Keywords: nucleation, nanowires, modeling, thermodynamics, growth, step-flow kinetics

\title{
1. Introduction
}

Development of nanotechnologies provides new problems to the theory of phase transformations - nucleation, growth and coarsening in open nanosystems.

Step-flow kinetics is one of the newest experimentally investigated features of nanowire's growth [1-3] by VLS method. Nanowires grow by rapid increasing (few milliseconds) of the nanowire's height by the value of height of one monoatomic layer with great incubation time (several seconds) between these increases. It is shown for the II-VI or III-V compounds where solubility of one component in the liquid alloy is very low that nucleation statistics is self-regulated and corresponds to sub - Poissonian distribution of waiting times between nucleation events [3] (similar idea was also suggested in [4]). In contrast, the solubility of $\mathrm{Si}$ in $\mathrm{Au}$ is very high and one would expect that self-regulation mechanism will not work in such systems. Yet, nature may not meet these expectations (see below).

Growth of sufficiently thin nanowires takes place in the mononuclear regime: for the growth of one atomic layer of the nanowire it is necessary for one nucleus to appear [5]. So, here we will limit ourselves with nanosystems in which the nucleation events proceed one by one and probability of simultaneous nucleation and of coexistence of several nuclei is negligible. Experimental results show that nucleus appears on the edge of the nanowire, on the junction of three phases: liquid, solid and vapor [2]. For the Si nanowires grown in atmosphere of low pressure of disilane it is shown that growth rate is diameter independent [6]. In this case, adsorption processes on the surface of the liquid droplet make the main contribution to the growth rate.

The following peculiarities should be taken into account in this case:

1) "Feedback" via depletion: in nanovolume even one successful nucleation and subsequent monolayer growth may cause significant depletion of whole nanosystem with one of components [7], changing the conditions (driving force) for next nucleation. In other words, system should "recover" from a previous nucleation before trying the next one.

2) Each new nucleation event proceeds in non-steady-state conditions, under rising concentration of solute supplied by external flux. So, the time dependence of the driving force should be taken into account [8]. 


\section{Model}

Our main aim was to describe the process of layer by layer growth of nanowhisker with help of classical nucleation theory [13], at least for the realistic case of mononuclear regime. Successful nucleation of 2D islands to the new layer needs sufficient supersaturation of gold with silicon. On the other hand, after successful nucleation the new layer grows fast and takes several thousands of silicon atoms causing substantial depletion of gold droplet with silicon. (We will see below that "depletion" actually means just decrease of supersaturation, making the nucleation barrier too high for immediate next nucleation). If one takes liquid droplet as a hemisphere of radius $R$, then change of concentration $\Delta c_{\max }$ (molar fraction of silicon) due to attachment of one monolayer of atoms is about $\Delta c_{\max }=-\frac{\pi R^{2}}{(2 \pi / 3) R^{3}}=-\frac{3}{2} \frac{h}{R}$. Since monolayer thickness $h$ is typically few Angstroms, the concentration change can be up to 2 percents (for $R$ of about $20 \mathrm{~nm}$ ). "To recover" after depletion and to make a new successful nucleation attempt, the system needs time during which whisker stands still and droplet is gradually saturated by depositing atoms. 


\section{Figure 1 here}

Actually, theoretical scheme should depend on the hierarchy of characteristic times [14]. The total flux $J$ related to deposition flux density $j^{d e p}$ just by $J=j^{d e p} 2 \pi R^{2}$. First of all, one should compare the time between arrivals of new $\mathrm{Si}$ atoms to the droplet, $\tau_{f l u x}=1 / J=1 /$ ( $j^{d e p} 2 \pi R^{2}$ ), characteristic time of Si diffusion across the liquid golden droplet, $\tau_{d i f}=R^{2} / D$, and time of atomic layer growth covering all surface after successful nucleation of 2D island, $\tau_{\text {layer }}$. If $\tau_{f l u x} \gg \tau_{d i f}$, then we can apply thermodynamics for system "droplet plus 2D island" at fixed number of both $\mathrm{Au}$ and $\mathrm{Si}$ atoms considering $\mathrm{Si}$ concentration as uniform in the droplet. For nanowire (NW) radius about $20 \mathrm{~nm}$ and diffusivity of $\mathrm{Si}$ in liquid (Au,Si) alloy $D$ about $10^{-9} \mathrm{~m}^{2} \cdot \mathrm{s}^{-1}$, the critical value of depositing flux density is $j_{\text {crit }}{ }^{d e p} \approx 10^{21} \mathrm{at} \cdot \mathrm{m}^{-2} \mathrm{~s}^{-1}$.

We consider the possibility of stoichiometric 2D island nucleation (Fig.1) (with concentration $c_{i}=1$ for the case of pure Si nanowire growth) from the supersaturated liquid solution with average $\mathrm{Si}$ concentration $c$. For this we calculate the Gibbs free energy of the system $G(x)$ at different fixed number $N_{S i}$ of $\mathrm{Si}$ atoms in the droplet with $N_{A u}$ gold atoms (or fixed average concentration $\left.c=N_{S i} /\left(N_{A u}+N_{S i}\right)\right)$ and look for minimum of this dependence (Fig. 4a). There $x$ is a single parameter that characterizes size of the nuclei, nucleus shape is determined, as usual, by mechanical equilibrium of surface tensions and by the place of nucleation (details will be shown below).

\subsection{Nucleus shape}

In our model we consider heterogeneous nucleation with the nucleus that appears on the edge of the nanowire (Fig.2). There $O$ is the center of the nanowire's upper base, $O_{1}$ and $\mathrm{O}_{2}$ are the centers of the internal and external curvatures of the nucleus.

\section{Figure 2 here}

The shape of the nucleus corresponds to the contour TMPK, which is determined by the mechanical equilibrium:

$$
\left\{\begin{array}{c}
\gamma_{i \alpha} \cos \theta_{1}-\gamma_{i} \sin \theta_{2}=0 \\
\gamma_{\alpha}+\gamma_{i \alpha} \sin \theta_{1}-\gamma_{i} \cos \theta_{2}=0
\end{array} .\right.
$$

$\gamma_{i}, \gamma_{\alpha}$ are the surface tensions of silicon (i) and Au-Si liquid phase $(\alpha)$ and $\gamma_{i \alpha}$ is the interfacial tension between silicon and liquid phase.

From (1) we can find values of the angles $\theta_{1}$ and $\theta_{2}$ for known values of $\gamma_{i}, \gamma_{\alpha}$ and $\gamma_{i \alpha}$

$$
\begin{aligned}
& \theta_{1}=\arcsin \left(\frac{\gamma_{i}^{2}-\gamma_{i \alpha}^{2}-\gamma_{\alpha}^{2}}{2 \gamma_{\alpha} \gamma_{i \alpha}}\right) \\
& \theta_{2}=\arcsin \left(\frac{\gamma_{i \alpha}}{\gamma_{i}} \cos \theta_{1}\right)
\end{aligned}
$$

No experimental data appear to have been published on the surface tension of solid silicon $\left(\gamma_{i}\right)$. The evaluation of $\gamma_{i}$ by Eustathopoulos et al. [15], $\gamma_{i}=1,08 \mathrm{~J} . \mathrm{m}^{-2}$, is in good agreement with the evaluation done elsewhere by Naidich et al [16].

Naidich et al. [17] have measured the surface tension of liquid (Au,Si) alloys in the temperature range 364 to about $1700^{\circ} \mathrm{C}$. From their measurements, the surface tension of liquid alloys at $T=823 \mathrm{~K}$ can be given approximately by the relation $\gamma_{\alpha}=1-0,4 c_{S i}$. For a Sisaturated liquid solution at $823 \mathrm{~K}\left(c_{S i} \approx 0,26\right)$ the surface tension is $\gamma_{\alpha} \approx 0,9 \mathrm{~J} \cdot \mathrm{m}^{-2}$.

In another work, Naidich et al. [16] have studied the wetting of solid silicon by liquid Si-saturated (Au-Si) alloys in the temperature range 637 - 1573K. Their measurements show 
that solid silicon is well wetted by liquid ( $\mathrm{Au}, \mathrm{Si})$ alloys, the wetting contact angle $(\theta)$ being lower than about $50^{\circ}$ whatever the temperature and the alloy composition. By analogy with liquid (Au,Ge)/solid-Ge system (known surface tension of solid Ge), they took the ratio $\gamma_{i} / \gamma_{\alpha}$ $=1,24$. This assumption allows the authors to calculate interfacial tension $\gamma_{i \alpha}$ between solid silicon and $\mathrm{Si}$-saturated $(\mathrm{Au}, \mathrm{Si})$ alloys from experimental values of contact angle $(\theta)$ and surface tension of $(\mathrm{Au}, \mathrm{Si})$ alloys $\left(\gamma_{\alpha}\right)$ as a function of temperature. At $T=823 \mathrm{~K}$, the interfacial tension is $\gamma_{i \alpha} \approx 0,4 \mathrm{~J} . \mathrm{m}^{-2}$.

Note moreover that, in a study of anisotropy of solid Si-liquid (A1,Si) interfacial tension, Sens et al [18] have shown that the maximum anisotropy $\gamma_{i \alpha}(001) / \gamma_{i \alpha}(111)$ is about $10 \%$. For this reason and for sake of simplicity, in the following we will use constant values of interfacial tensions at $T=823 \mathrm{~K}$ :

$\gamma_{\alpha} \approx 0,9 \mathrm{~J} \cdot \mathrm{m}^{-2}, \gamma_{i}=1,08 \mathrm{~J} \cdot \mathrm{m}^{-2}$, and $\gamma_{i \alpha} \approx 0,4 \mathrm{~J} \cdot \mathrm{m}^{-2}$.

In our model we vary the value of $H L=x$ which allows us to fix the both edges of the internal $l_{1}$ (contour TMP) and the external $l_{2}$ (contour TKP) curvatures of the nucleus. We denote the origin curvature of the nanowire bounded by these edges as $l$ (contour $T L P$ ). For the small values of $x$ in comparison with the radius of the nanowire $R$ we can evaluate the length of all curvatures and the area of the nucleus:

$$
\begin{aligned}
& l=2 \sqrt{2 R x}, \quad x<<R \\
& l_{1}=\frac{2\left(\pi / 2-\theta_{1}\right) \sqrt{2 R x}}{\cos \theta_{1}}, \\
& l_{2}=\frac{2 \theta_{2} \sqrt{2 R x}}{\sin \theta_{2}}, \\
& S=2 R x \varepsilon
\end{aligned}
$$

when $\varepsilon$ depends only on $\theta_{1}$ and $\theta_{2}$ :

$$
\varepsilon=\frac{\left(\pi / 2-\theta_{1}-\sin \theta_{1} \cos \theta_{1}\right)}{\cos ^{2} \theta_{1}}+\frac{\left(\theta_{2}-\sin \theta_{2} \cos \theta_{2}\right)}{\sin ^{2} \theta_{2}}
$$

Now we can write the dependence between $x$ and the number of atoms in the nucleus $n$ (from eq. (5) and $S=n \Omega / h$ ):

$$
x=\frac{n \Omega}{2 R h \varepsilon}
$$

here $\Omega-$ is the atom volume.

A calculation for reasonable parameters shows that radius $\mathrm{O}_{1} \mathrm{M}$ of internal boundary $l_{1}$ increases more rapidly than radius $\mathrm{O}_{2} \mathrm{~K}$ of external boundary $l_{2}$, so for the small nucleus (consisting of few tens of silicon atoms) we can neglect the deviation of external boundary from initial circle, and, respectively, neglect the distortion of the droplet surface in the vicinity of the nucleation site.

\subsection{Thermodynamics of nucleation and depletion in nanodroplet}

\section{a) Driving forces.}

Change of Gibbs potential of the system at conditions of epitaxy can be defined as:

$$
\Delta G=n \Delta g^{\text {bulk }}+\Delta G^{\text {surf }}=n \Delta g^{\text {bulk }}+\gamma_{i a} l_{l} h+\gamma_{i} l_{2} h-\gamma_{\alpha} l h \text {, }
$$

here $h$ is the height of the monolayer, $\Delta g^{\text {bulk }}$ is the bulk driving force per one atom of the nucleus (it is negative).

$$
\Delta g^{b u l k}=N / n[g(c-\Delta c)-g(c)]+g_{i}-g(c-\Delta c)
$$

here $g(c)$ is the Gibbs free energy per one atom of the liquid phase $(\alpha)$ with concentration $c, g_{i}$ is the Gibbs free energy per one atom of the solid phase with concentration $c_{i}=1$ (pure 
silicon), $\Delta c$ is the concentration depletion after nucleus appearing, $N-$ total amount of the atoms in droplet.

Here we use experimental data and thermodynamic assessment of Au-Si system [19,20] (see Fig.3 for the phase diagram). The Au-Si solution is modelled as liquid solution, in which the Gibbs energies are expressed as $G=\sum_{i=A u, S i} c_{i} G_{i}^{0}+R T \sum_{i=A u, S i} c_{i} \ln c_{i}+G^{E}$, where $G_{i}^{0}$ - is the Gibbs energies of pure element of $\mathrm{Au}$ and $\mathrm{Si}$ at each temperature from [19] (which are reference states for $\mathrm{Au}$ and $\mathrm{Si}$ ), $G^{E}$ - is the excess Gibbs energy, expressed by model of subregular solution [20]. In particular, at $T=823 \mathrm{~K}$, the expression of $G$ is given by the following equation:

$$
\begin{gathered}
G[\mathrm{~J} / \mathrm{mole}]=6702.37 \cdot c-36320.01 \cdot(1-c)+6842.42 \cdot\{c \cdot \ln (c)+(1-c) \cdot \ln (1-c)\} \\
-c \cdot(1-c)\{36562.56+28464.63 \cdot(1-2 c)\}
\end{gathered}
$$

\section{Figure 3 here}

\section{b) Equilibrium conditions.}

We take into account the possible depletion of the droplet by the very process of 2Dnucleation. In our conditions heterogeneous nucleation occurs in volume of metastable phase of gold-silicon liquid solution. Depending on the number of $\mathrm{Si}$ atoms in the droplet the following situations are possible, typical for nanoparticles and nanosize diffusion zones, see for example [7,21,22]:

1) At sufficiently small number of Si atoms (relatively small $c$ ) Gibbs's free energy of the droplet has only one minimum corresponding to zero (curve (1) at Fig. 4b);

2) At larger number of $\mathrm{Si}$ atoms dependence $G(x)$ becomes non-monotonic with second metastable minimum (curve (2) at Fig. 4b);

3) At some critical number of $\mathrm{Si}$ atoms second minimum becomes stable (curve (3) at Fig. 4b);

After this the formation of new monolayer becomes thermodynamically favoured but kinetically it depends on the height of nucleation barrier. This height becomes smaller and smaller with arriving of new silicon atoms inside droplet. It means that to predict the growth characteristics we must modify Zeldovich steady-state nucleation model [13] for the case of non-stationary driving force.

\section{Figure 4 here}

\section{c) Capillary effects}

Capillary effects are usually treated in terms of curvature dependent Laplace pressure. It is appropriate in case of spherical or cylindrical surface of the nucleus, otherwise direct calculation of Laplace pressure near curved interfaces may be misleading. Actually, the capillary effect will cause the size dependence of Gibbs energy, chemical potential and of corresponding equilibrium composition. It is convenient instead of introducing individual curvatures for the different sides of the nucleus to use a single effective curvature $k^{e f}=1 / R^{e f}$, $R^{e f}$ is the effective radius of curvature which will be introduced below.

Stable or unstable equilibrium (including saddle-point at the nucleation barrier) is determined by the condition of zero derivative of change of Gibbs energy $\Delta G$ (see eq. (8)):

$$
\begin{aligned}
& \frac{d \Delta G}{d x}=\Delta g^{\text {bulk }}+\frac{d \Delta G^{\text {surf }}}{d n}=\Delta g^{\text {bulk }}+h\left(\gamma_{i \alpha} \frac{d l_{1}}{d n}+\gamma_{i} \frac{d l_{2}}{d n}-\gamma_{\alpha} \frac{d l}{d n}\right) \\
& =\Delta g^{\text {bulk }}+h \frac{d x}{d n}\left(\gamma_{i \alpha} \frac{d l_{1}}{d x}+\gamma_{i} \frac{d l_{2}}{d x}-\gamma_{\alpha} \frac{d l}{d x}\right)
\end{aligned}
$$

here $\gamma^{e f}$ - is the effective surface energy coefficient (combination of $\gamma_{i \alpha}, \gamma_{i}$ and $\gamma_{\alpha}$, see below). We can find derivatives from (2-4) and (7): 


$$
\frac{d l}{d x}=\sqrt{\frac{2 R}{x}}, \quad \frac{d l_{1}}{d x}=\frac{\left(\pi / 2-\theta_{1}\right) \sqrt{2 R}}{\cos \theta_{1} \sqrt{x}}, \quad \frac{d l_{2}}{d x}=\frac{\theta_{2} \sqrt{2 R}}{\sin \theta_{2} \sqrt{x}}, \quad \frac{d x}{d n}=\frac{\Omega}{2 R h \varepsilon}
$$

Hence,

$$
\frac{d \Delta G}{d x}=\Delta g^{\text {bulk }}+\frac{\Omega}{\sqrt{2 R x} \varepsilon} \gamma^{e f}=\Delta g^{\text {bulk }}+\frac{\text { const }}{\sqrt{n}}=\Delta g^{\text {bulk }}+g_{\text {capillar }}(n)
$$

with $\quad \gamma^{e f}=\frac{\left(\pi / 2-\theta_{1}\right)}{\cos \theta_{1}} \gamma_{i \alpha}+\frac{\theta_{2}}{\sin \theta_{2}} \gamma_{i}-\gamma_{\alpha}$.

Here $\quad g_{\text {capillar }}(n)=\frac{\partial \Delta G^{\text {surf }}}{\partial n}=\frac{\gamma^{e f} \Omega}{R^{e f}(n)}$

with $R^{e f}(n)=\sqrt{2 R x} \varepsilon$

Thus the concentration of silicon in the droplet in equilibrium with 2D island of size $n$ at the edge of nanowire surface may be found using standard common tangent construction (Fig. 4b) with rising the Gibbs energy $g_{i}$ of the pure silicon by "Laplace term" $g_{\text {capillar }}(n)$.

The change of the value of equilibrium composition $\delta c=c_{e q}(n)-c_{e q}$ (here $c_{e q}$ corresponds to equilibrium composition on the planar boundary) due to the appearing of curvatures on the nucleus shape should be found by using of common tangent rule (Fig.4b) is:

$$
\delta c=c_{e q}(n)-c_{e q}=\frac{g_{\text {capillar }}(n)}{(1-c) g^{\prime \prime}}
$$

here $g^{\prime \prime}$ is the curvature of the dependence $g_{\alpha}(c)$ in point $c_{e q}$. From (15) we obtain:

$$
\delta c=c_{e q}(n)-c_{e q}=\frac{g_{\text {capillar }}(n)}{(1-c) g^{\prime \prime}}=\frac{\gamma^{e f} \Omega}{\sqrt{2 R x} \varepsilon} \frac{1}{(1-c) g^{\prime \prime}}
$$

\subsection{Kinetics}

Let's consider kinetic model of nucleation and lateral growth of nanowhisker taking into account deposition flux $j^{d e p}$ of silicon atoms (in atoms of Si per $\mathrm{m}^{2}$ of liquid surface) from disilane gas [23]:

$$
j^{d e p}=\frac{P}{4 k T} \sqrt{\frac{8 k T}{\pi m_{d i s s}}}
$$

here $P$ is the disilane pressure, $m_{\text {diss }}$ is the mass of disilane molecule $\left(\mathrm{Si}_{2} \mathrm{H}_{6}\right)$.

In the following the molar fraction of silicon $c_{S i}=N_{S i} /\left(N_{S i}+N_{A u}\right)$ will be noted by $c$.

Conservation law for silicon atoms in the droplet before nucleation gives:

$$
\frac{d N_{S i}}{d t}=2 j^{d e p} \pi R^{2}
$$

The rate of change of mean concentration of silicon in the droplet before the next nucleation event is approximately equal to:

$$
\frac{d c(t)}{d t}=\frac{(1-c(t))}{N_{S i}+N_{A u}} \frac{d N_{S i}}{d t}=(1-c(t)) \frac{3 \Omega}{R} j^{d e p}
$$

We can represent change of mean concentration of Si discretely:

$$
c(t+d t)=c(t)+(1-c(t)) \frac{3 \Omega}{R} j^{d e p}
$$

Nucleation frequency can be defined as:

$$
v(t)=e^{-\tau / t} \cdot s\left(G^{*}, x_{c r}\right) \cdot 2 \pi R
$$

here $\tau$ is a lag-time, necessary to reach the critical size by random walk in the size space without influence of nucleation barrier, $s$ - steady-state flux in the size space (number of 
islands intersecting critical size per unit time per unit length of liquid/solid/vapor junction with the total length $2 \pi R$ ) is taken from Zeldovich theory modified for time-dependent nucleation on the one-dimensional contour, nucleus size being determined by characteristic length $x$ at Fig.2:

$$
s=\sqrt{\frac{-\Delta G^{\prime \prime}}{\pi k T}} f^{e q}\left(x_{c r}\right) B\left(x_{c r}\right)
$$

here $\Delta G^{\prime \prime}=\left(\frac{d^{2} \Delta G}{d x^{2}}\right)_{x=x_{c r}}$ - is the curvature of the nucleation barrier, $f\left(x_{c r}\right)$ - equilibrium size distribution, and diffusivity of nucleus in the size space near critical size $B\left(x_{c r}\right)$ is given by:

$$
\begin{aligned}
& B(x)=-k T \frac{\left(\frac{d x}{d t}\right)_{x \rightarrow x_{c r}}}{\left(\frac{d \Delta G}{d x}\right)_{x \rightarrow x_{c r}}} \\
& f^{e q}\left(x_{c r}\right)=\text { const. } \exp \left(\frac{-\Delta G\left(x_{c r}\right)}{k T}\right)
\end{aligned}
$$

here $\Delta G\left(x_{c r}\right)=\Delta G^{*}$ is a nucleation barrier which previously (in Zeldovich theory) was treated as constant in time.

As we have a nucleus of pure silicon, hence, each silicon atom on the triple junction could be the centre of nucleation. So, the value of const in eq.(19) can be found according to receipt [24]:

$$
\text { const }=\frac{c}{\Omega^{1 / 3}} \frac{d n}{d x}
$$

Indeed, if the nucleus size is characterized by number of atoms then $f(n=1)$ is the number of silicon atoms at the perimeter $2 \pi R$, equal to $c \cdot\left(2 \pi R / \Omega^{1 / 3}\right)$, divided by the length of that perimeter.

$$
f^{e q}(n=1)=\frac{c}{\Omega^{1 / 3}} .
$$

If, instead of $n$, nucleus size is characterized by length $x$ then, the distribution functions are transformed in the standard way:

$$
f(x)=\frac{d n}{d x} f(n)
$$

which immediately gives eq. (25).

\section{Determination of "diffusivity in size space" $B\left(x_{c r}\right)$}

a) Calculation of $\left(\frac{d x}{d t}\right)_{x \rightarrow x_{c r}}$

In this paper we will limit ourselves to diffusion-controlled nucleation. Interface controlled nucleation will be considered elsewhere. Critical radius (typically about $1 \mathrm{~nm}$ ) is assumed to be much smaller than the nanowire radius (typically ten nanometers or more), so that 2D shape of nucleus does not prevent concentration distribution to be almost spherically symmetrical. At first we use the conservation of matter considering 2D island surrounded by sphere of radius sufficiently large, so that in this radius almost hemispherical symmetry can be assumed.

Relation between total diffusion flux $J^{\text {dif }}$ in the radial direction and the rate of nucleus growth (unusual combination of 3D diffusion with 2D particle seems $\mathrm{OK}$ as far as particle size is much smaller than the droplet size): 


$$
-J^{d i f}=\frac{d}{d t}\left(\frac{S h}{\Omega}\right)=\frac{h}{\Omega} \frac{d x}{d t} \frac{d S}{d x},
$$

with $\quad J^{d i f}=\eta j(\rho) 2 \pi \rho^{2}$ at any $\rho>>x$.

Factor $\eta$ characterizes the deviation from the spherical geometry in the vicinity of nucleus. Unfortunately, we cannot suggest anything better than analytic solution under spherical geometry and roughly assume $\eta \approx 1 / 2$.

As shown in textbooks on Ham's model of precipitate growth in 3D space or for 3Dripening [25], the steady state flux density in 3D space is:

$$
j(\rho)=-\frac{D\left(c-c_{e q}(n)\right)}{\Omega \rho^{2}} r_{\text {min }}^{e f}
$$

here $r_{\text {min }}^{e f}=\sqrt{S / \pi},(S$ is determined by eq. (5))

Moreover, from eqs. (7) and (21) we obtain:

$$
\frac{d S}{d x}=2 R \varepsilon
$$

and $\quad c-c_{e q}(n)=c-c_{e q}-\frac{\gamma^{e f}}{\sqrt{2 R x} \varepsilon} \frac{1}{(1-c) g^{\prime \prime}}$

From combination of (26-30) we obtain the growth rate:

$$
\frac{d x}{d t}=\sqrt{\frac{2 \pi}{R \varepsilon}} \frac{\eta D\left[\left(c-c_{e q}\right) \sqrt{x}-\frac{\Omega}{\sqrt{2 R}(1-c) g^{\prime \prime}} \frac{\gamma^{e f}}{\varepsilon}\right]}{h}
$$

Zero value of the growth rate corresponds to the critical size. Thus,

$$
\frac{\Omega}{\sqrt{2 R}(1-c) g^{\prime \prime}} \frac{\gamma^{e f}}{\varepsilon}=\left(c-c_{e q}\right) \sqrt{x_{c r}} .
$$

Hence,

$$
\left(\frac{d x}{d t}\right)_{x \rightarrow x_{c r}}=\sqrt{\frac{2 \pi}{R \varepsilon}} \frac{\eta D\left(c-c_{e q}\right)\left(\sqrt{x}-\sqrt{x_{c r}}\right)}{h}
$$

b) Calculation of $\left(\frac{d \Delta G}{d x}\right)_{x \rightarrow x_{c r}}$

From (12), with taking into account (11) and (29), we obtain:

$$
\frac{d \Delta G}{d x}=\frac{d \Delta G}{d n} \frac{d n}{d x}=\left(\Delta g^{b u l k}+\frac{\Omega \gamma^{e f}}{\varepsilon \sqrt{2 R x}}\right) \frac{2 R h \varepsilon}{\Omega}=\Delta g^{b u l k} \frac{2 R h \varepsilon}{\Omega}+\frac{h \gamma^{e f} \sqrt{2 R}}{\sqrt{x}}
$$

Critical radius of two-dimensional nucleus corresponds to zero derivative of Gibbs energy change, thus, $\Delta g^{\text {bulk }} \frac{2 R h \varepsilon}{\Omega}=-\frac{h \gamma^{e f} \sqrt{2 R}}{\sqrt{x}}$. Hence,

$$
\left(\frac{d \Delta G}{d x}\right)_{x \rightarrow x_{c r}}=h \gamma^{e f} \sqrt{2 R}\left(\frac{1}{\sqrt{x}}-\frac{1}{\sqrt{x_{c r}}}\right)=h \gamma^{e f} \sqrt{2 R}\left(\frac{\sqrt{x_{c r}}-\sqrt{x}}{x_{c r}}\right)
$$

Finally, after substitution of (33) and (35) in (23), we obtain:

$$
B\left(x_{c r}\right)=-k T \sqrt{\frac{2 \pi}{R \varepsilon}} \frac{\eta D\left(c-c_{e q}\right)\left(\sqrt{x}-\sqrt{x_{c r}}\right)}{h} \cdot \frac{x_{c r}}{h \gamma^{e f} \sqrt{2 R}\left(\sqrt{x_{c r}}-\sqrt{x}\right)}=k T \sqrt{\frac{\pi}{\varepsilon}} \frac{\eta x_{c r} D\left(c-c_{e q}\right)}{R h^{2} \gamma^{e f}}
$$

Or, for escape from simultaneous presence of supersaturation term $\left(c-c_{e q}\right)$ and critical size parameter $x_{c r}$ we can use (32) once again: 


$$
\begin{gathered}
B\left(x_{c r}\right)=-k T \sqrt{\frac{2 \pi}{R \varepsilon}} \frac{\eta D\left(c-c_{e q}\right)\left(\sqrt{x}-\sqrt{x_{c r}}\right)}{h} \cdot \frac{x_{c r}}{h \gamma^{e f} \sqrt{2 R}\left(\sqrt{x_{c r}}-\sqrt{x}\right)}=k T \sqrt{\frac{\pi}{\varepsilon}} \frac{\eta x_{c r} D\left(c-c_{e q}\right)}{R h^{2} \gamma^{e f}} \\
B\left(x_{c r}\right)=k T \sqrt{\pi / 2} \frac{\eta D \Omega}{R^{3 / 2} \varepsilon^{3 / 2} h^{2}(1-c) g^{\prime \prime}} \sqrt{x_{c r}}
\end{gathered}
$$

We determine nucleation probability and then use standard Monte Carlo scheme with eq. (21):

$$
p(\Delta t)=v(t) \Delta t,
$$

If, at some value $t^{*}$, random $<p(\Delta t)$, then $\bar{c}_{o, i+1}=\bar{c}_{i}\left(t^{*}\right)-\frac{3 h}{2 R}$

\section{Parameters and basic algorithm for modeling of step-wise nanowire growth.}

Disilane pressure was changed between: $1 \cdot 10^{-7}-3 \cdot 10^{-5}$ Torr (typical values used in Refs. $[1,2])$, Diffusivity of Si in liquid solution $D=10^{-9} \mathrm{~m}^{2} / \mathrm{s}$, Height of monolayer $h=0.31 \cdot 10^{-9} \mathrm{~m}$,

Radius of liquid droplet (taken here approximately as hemispherical) $R=22 \mathrm{~nm}$, Time-step was chosen depending on flux density, as one percent of anticipated average waiting time: $d t_{i}=\frac{\langle t\rangle_{i}}{100}=\frac{h}{100 \cdot \Omega j_{i}^{d e p}}$;

A formulated model was used for numeric calculation of time behavior of supersaturation and step-flow kinetics. At that, Monte Carlo algorithm was used.

\section{Results and discussion}

We cannot claim to predict the mean waiting time in the steady-state regime: evidently, this average time is just an inverse total flux $J\left(<t>=\frac{h}{\Omega j^{d e p}}\right)$. Yet, one can suggest other characteristics. Typical time dependences of nucleation probability and of silicon content in gold droplet are presented on Fig.5. In particular, Fig.5b demonstrates that system possesses elements of self organization - the concentration of silicon in the droplet soon "forgets" about its initial value and fluctuates around some steady-state asymptotic value determined by the magnitude of incoming flux: the larger is the flux, the higher is the average supersaturation. Dependence of asymptotic average value of composition on incoming flux is shown in Fig.6. Time correlations between these fluctuations are discussed below.

\section{Figure 5 here}

In Figure $5 \mathrm{~b}$ one can also see that supersaturation remains significantly larger than the depletion during one monolayer growth. Indeed, the variation of silicon concentration in the drop after nucleation and "instantaneous" growth of a silicon monolayer, for $h=0,31 \mathrm{~nm}$ and $R=22 \mathrm{~nm}, \Delta c_{\max }=0,0211$ is twice lower than the mean silicon supersaturation $\bar{c}-c_{e q}$ of about 0,046 . Thus, DEPLETION as a result of nucleation, in our previous considerations means just lower supersaturation with respectively high nucleation barrier.

Fig.6 shows that the inverse of the supersaturation decreases linearly with the logarithm of the incoming flux density (proportional to disilane pressure). This linear character of dependence can be explained by a rather simple theoretical model to be presented elsewhere. 


\section{Figure 6 here}

In table 1 values of nucleation barriers energies for average silicon concentration (as well as the minimum and maximum values) are presented for different values of incoming flux. In this table values of average supersaturations are also reported. It is seen that the nucleation barrier energy decreases with the increasing incoming flux which is in agreement with the fact that silicon supersaturation increases with the deposition flux (see Fig. 6a).

In the following tables and plots we present:

Average waiting time: $\left\langle t>=\frac{\sum_{i=1}^{n} t_{i}}{n}\right.$

Amount of intervals (successful nucleations) for each calculation is $n=1000$.

Square root of average squared deviation of waiting times: $\Delta=\sqrt{\frac{\sum_{i=1}^{n}\left(t_{i}-<t>\right)^{2}}{n-1}}$

Dimensionless standard deviation: $d=\frac{\Delta}{<t>}$

Absolute time correlation: $C=\frac{\sum_{i=2}^{n}\left(t_{i}-<t>\right)\left(t_{i-1}-<t>\right)}{n-1}$

Dimensionless time correlation: $c=\frac{C}{\Delta}=\frac{\overline{\left(t_{i}-<t>\right)\left(t_{i-1}-<t>\right)}}{\overline{\left(t_{i}-<t>\right)^{2}}}$

Fig.7 gives the variation of dimensionless standard deviation and dimensionless time correlation on the logarithm of deposition flux density. The dimensionless standard deviation increases almost proportionally with the logarithm of the disilane pressure (in Torr) up to $1 \cdot 10^{-5}$ Torr whereas the variation of dimensionless time correlation presents an asymptotic behavior (i.e. for high flux densities it tends to zero).

\section{Figure 7 here}

Distributions for the disilane pressure $P=4 \cdot 10^{-7}, 5 \cdot 10^{-6}$ and $3 \cdot 10^{-5}$ Torr are presented on the Fig.8. They are rather well fitted by Weibull distribution. Parameters of Weibull distribution function $\left(y=f(x \mid a, b)=b a^{-b} x^{b-1} e^{-\left(\frac{x}{a}\right)^{b}} I_{(0, \infty)}(x)\right)$ are estimated. The larger is $\mathrm{b}$, the narrower is distribution.

\section{Figure 8 here}

\section{Summary}

1. Possibility of jerky motion is confirmed in the model using assumptions of heterogeneous nucleation, mononuclear mechanism and diameter independent growth rate.

2. Zeldovich nucleation theory (including determination of diffusivity in the size space) is adapted for the case of: a) heterogenous nucleation; b) complex geometry; c) timedependent driving force.

3. Asymptotic supersaturation of liquid Au with Si increases with increasing incoming flux. Inverse supersaturation is a linear descending function of the flux density logarithm. 
4. Standard deviation of reduced waiting times distribution increases with increasing deposition flux. It correlates with time correlation for subsequent monolayers. Stronger time correlation corresponds to narrower waiting time distribution. This result obtained for silicon repeats conclusion of [3] for VLS growth of GaAs nanowhiskers.

5. Waiting time correlation for subsequent events is negative and it increases by absolute value with decreasing incoming flux. Namely, the absolute value of the dimensionless time correlation is approximately inversely proportional to the flux density.

6. Waiting time distribution is well fitted by Weibull plots with standard deviation decreasing with decreasing flux density.

\section{Acknowledgment}

Work was supported by the DNIPRO program (EGIDE - France and Ministry of Education and Science of Ukraine) and also by Ukrainian State Fund for Fundamental Research.

\section{References}

[1] C.Y. Wen, J. Tersoff, M.C. Reuter, E.A. Stach and F.M. Ross, Phys. Rev. Lett. 105 (2010) p. 195502

[2] F.M. Ross, Rep. Progr. Phys. 73 (2010) p. 114501

[3] F. Glas, J-C. Harmand and G. Patriarche. Phys. Rev. Lett. 104, (2010) p. 135501

[4] A. Gusak, A. Kovalchuk and K.N. Tu, PTM-2010, Avignon, France (2010), p. 91. Available at http://www.ffc-asso.fr/PTM2010/fichs/doc/PTM2010-Recueil_COMPLET31-05-2010.pdf

[5] D. Kashchiev, Cryst. Growth Des. 6 (2006) p. 1154

[6] S. Kodambaka, J. Tersoff, M.C. Reuter and F.M. Ross, Phys. Rev. Lett. 96 (2006) p. 096105

[7] A.S. Shirinyan and A.M. Gusak, Phil. Mag. A 84 (2004) p. 579

[8] D. Kashchiev, Surf. Sci. 14 (1969) p. 209

[9] A.O. Kovalchuk, A.M. Gusak and K.N. Tu, Nano Lett. 10 (2010) p. 4799

[10] K-C Lu, K.N. Tu, W.W. Wu, L.J. Chen, B-Y Yoo and N.V. Myung, Appl. Phys. Lett. 90 (2007) p. 253111

[11] Y-C Lin, K-C Lu, W-W Wu, J. Bai, L.J. Chen, K.N. Tu and Y. Huang, Nano Lett. 8 (2008) p. 913

[12] Y-C Chou,W-W Wu, L-J Chen and K-N Tu. Nano Lett. 9 (2008) p. 2337

[13] L.D. Landau and E.M. Lifshitz, Physical Kinetics, Vol. 10, Pergamon, London, 1981

[14] V. G. Dubrovskii, N. V. Sibirev, J. C. Harmand and F. Glas, Phys. Rev. B 78 (2008) p. 235301

[15] N. Eustathopoulos, M. Nicholas, B. Drevet, Wettability at high temperatures, Pergamon Materials Series, v.3, Pergamon, Oxford, 1999

16. Y.V. Naidich, V.M. Perevertailo and L.P. Obushchak, Poroshkavaya Metall. 5 (1975) p. 73

[17] Y.V. Naidich, V.M. Perevertailo and L.P. Obushchak, Poroshkavaya Metall. 7 (1975) p. 63

[18] H. Sens and N. Eustathopoulos, J. Cryst. Growth 98 (1989) p. 751

[19] A.T. Dinsdale, Calphad 15 (1991) p. 317

[20] F.G.Meng, H.S. Liu, L.B. Liu and Z.P. Jin, J. Alloys Comp. 431 (2007) p. 292

[21] F. Hodaj, A. Gusak, Acta Mat. 52 (2004) p. 4305

[22] A.M. Gusak, Diffusion-controlled Solid State Reactions, WILEY-VCH, Weinheim, 2010 
[23] K.N. Tu, Electronic Thin-Film Reliability, Cambridge University Press, Cambridge, 2010

[24] V.V. Slezov, J. Schmelzer J. Phys. Chem. Solids 55 (1994) 243

[25] P. Shewmon, "Diffusion in solids", TMS, Warrendale, 1992 


\section{Figure captions}

Figure 1. Model system for nanowire

Figure 2. Shape of the nucleus and the directions of the surface tensions

Figure 3 The Au-Si phase diagram calculated in [20]

Figure 4. a) Dependence of thermodynamic Gibbs potential of the system during nucleation; b) Model Gibbs potentials of liquid solution and silicon (schematically);

Figure 5. Typical nucleation probability dependence on time (a) and typical silicon concentration dependence on time (b) for Si nanowires with $R=22 \mathrm{~nm}$ grown at $T=823 \mathrm{~K}$ and disilane pressure $4 \cdot 10^{-7}$ Torr.

Figure 6. Dependence of inverse supersaturation on the logarithm of disilane pressure (in Torr) for Si nanowires with $R=22 \mathrm{~nm}$ grown at $T=823 \mathrm{~K}$.

Figure 7. Dependences of dimensionless standard deviation (a) and the logarithm dimensionless time correlation (b) on the logarithm of disilane pressure (In Torr). Note that in (b) the gradient is close to -1 which means that the absolute value of the dimensionless time correlation is approximately inversely proportional to the flux density. Phase-plane portrait of dimensionless standard deviation and dimensionless time correlation is presented (c).

Figure 8. Distribution of waiting times fitted by Weibull distribution function: for the disilane pressure $4 \cdot 10^{-7}$ Torr with parameters $a=1,045, b=10,526$ (a); for the disilane pressure $5 \cdot 10^{-6}$ Torr with parameters $a=1.052, b=8,697(\mathrm{~b})$; for the disilane pressure $3 \cdot 10^{-5}$ Torr with parameters $a=1,064, b=7,030$ (c). 


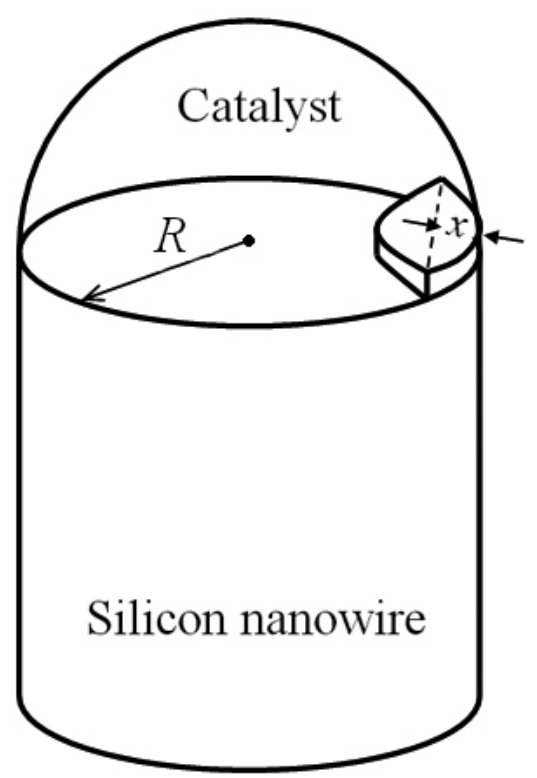

Figure 1 


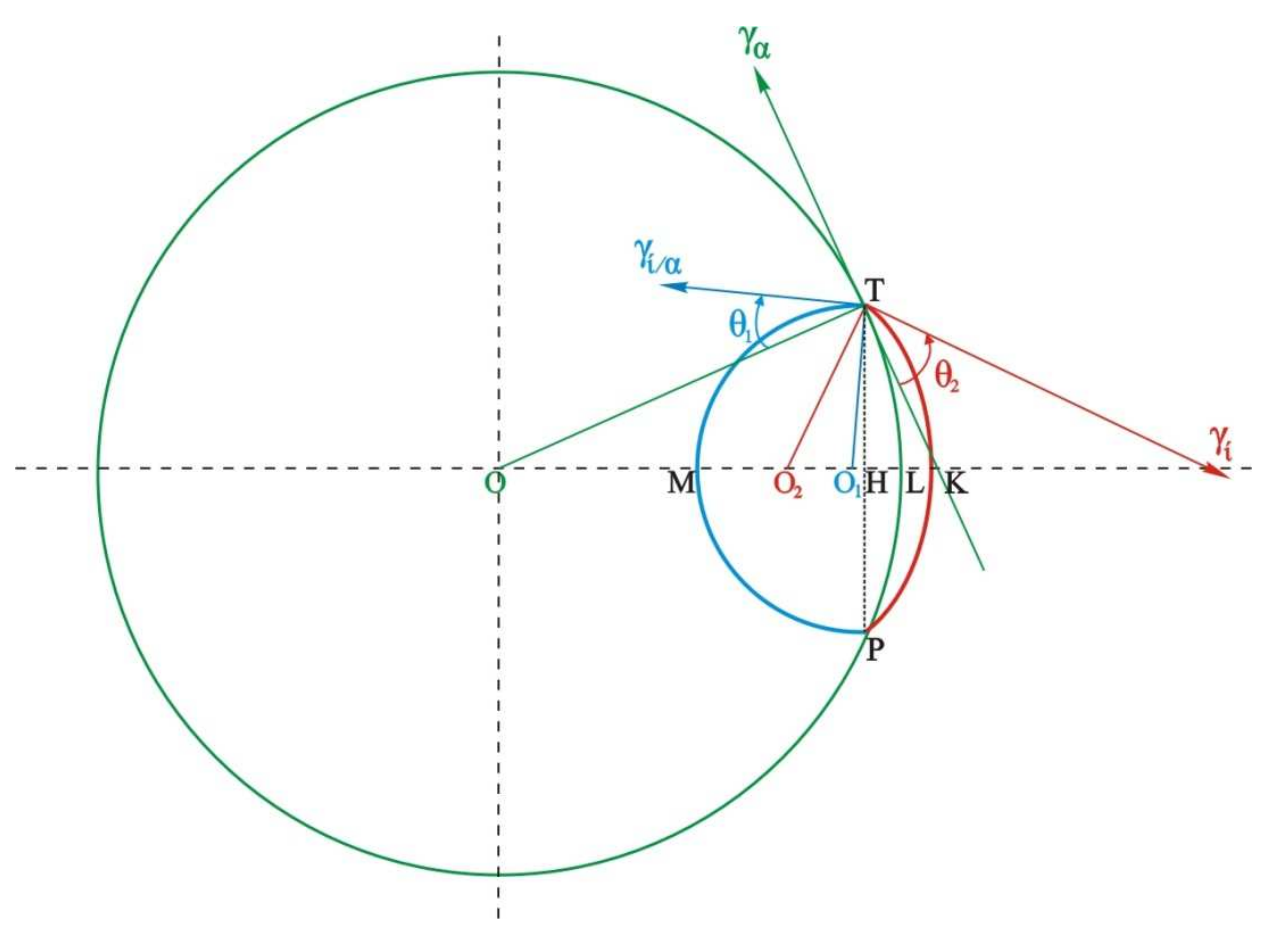

Figure 2

http://mc.manuscriptcentral.com/pm-pml 


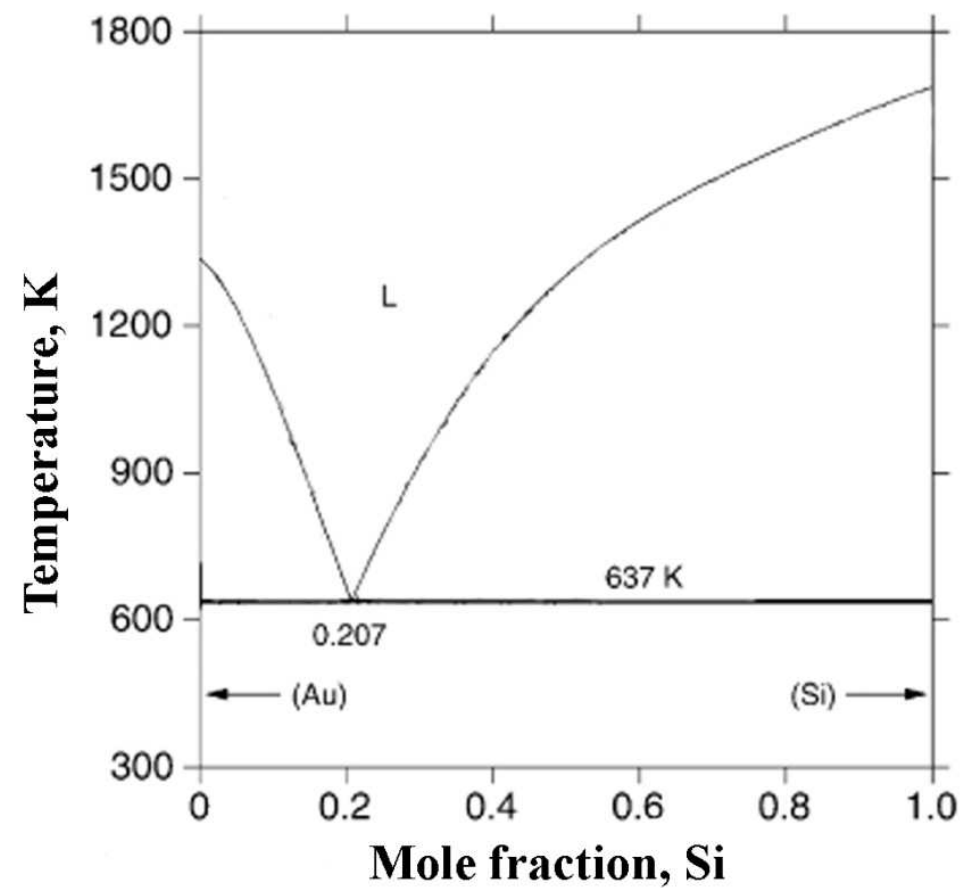

Figure 3 

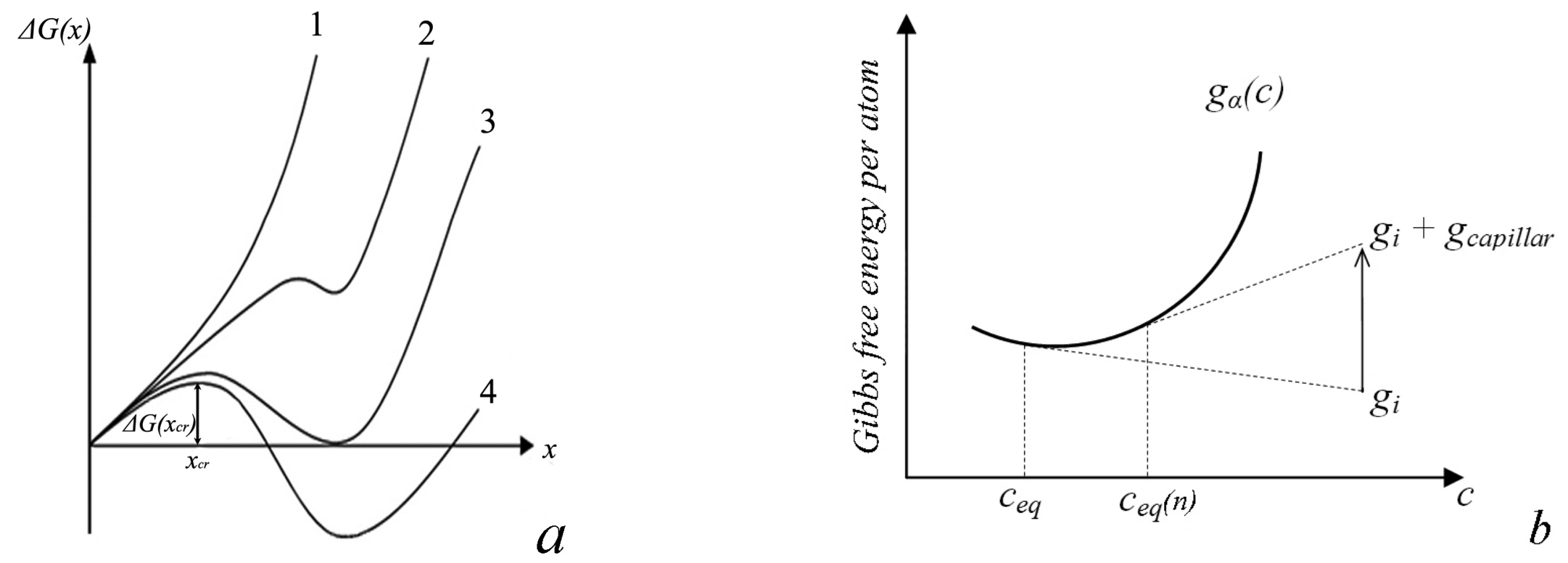

Figure 4 

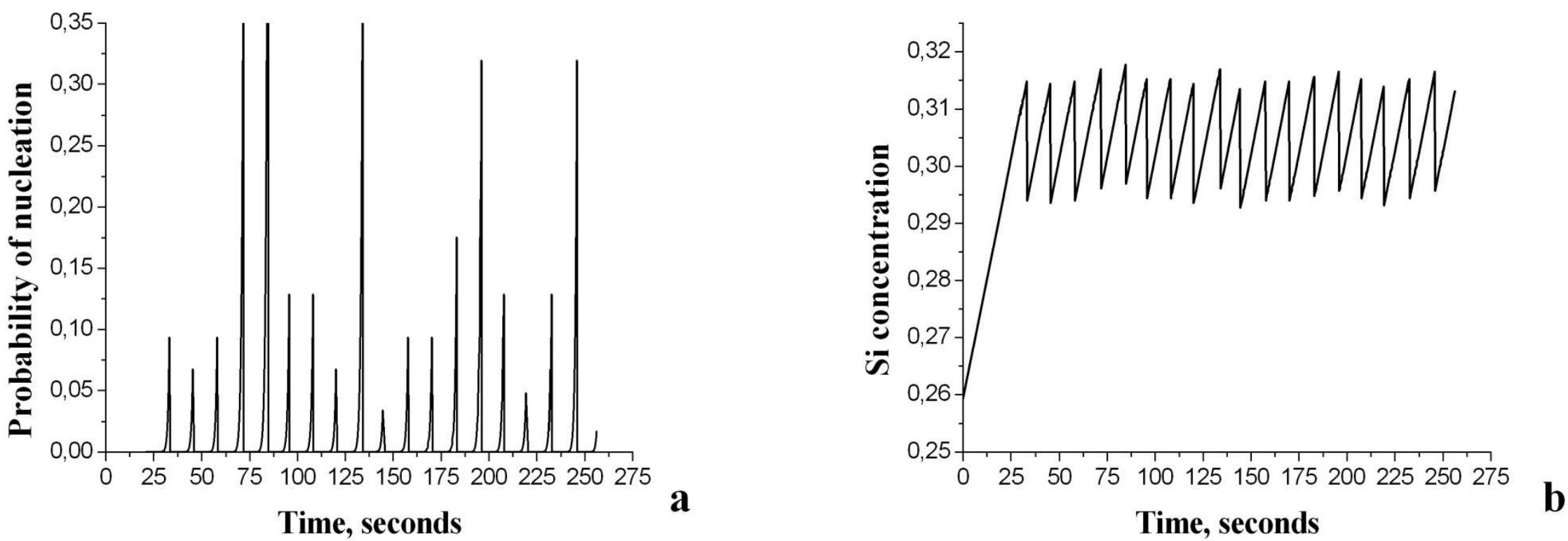

Figure 5 


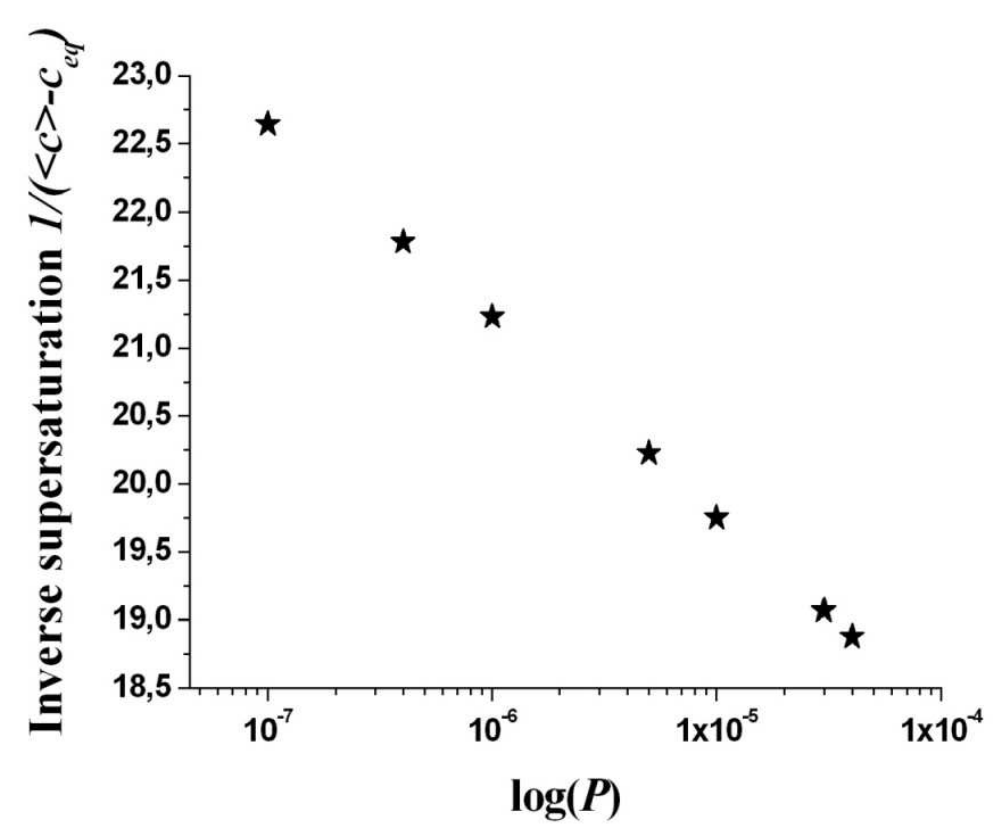

Figure 6 

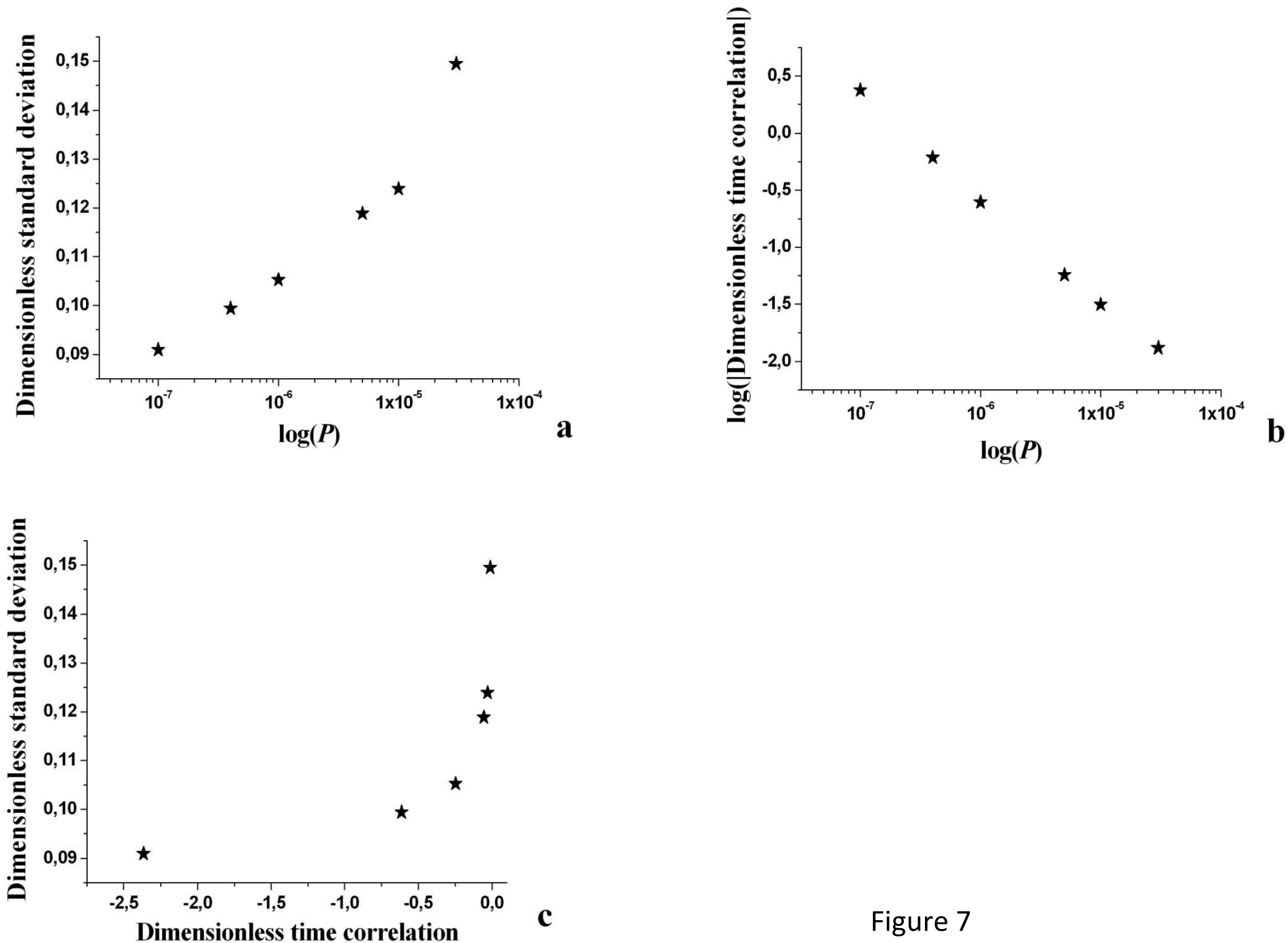

Figure 7 

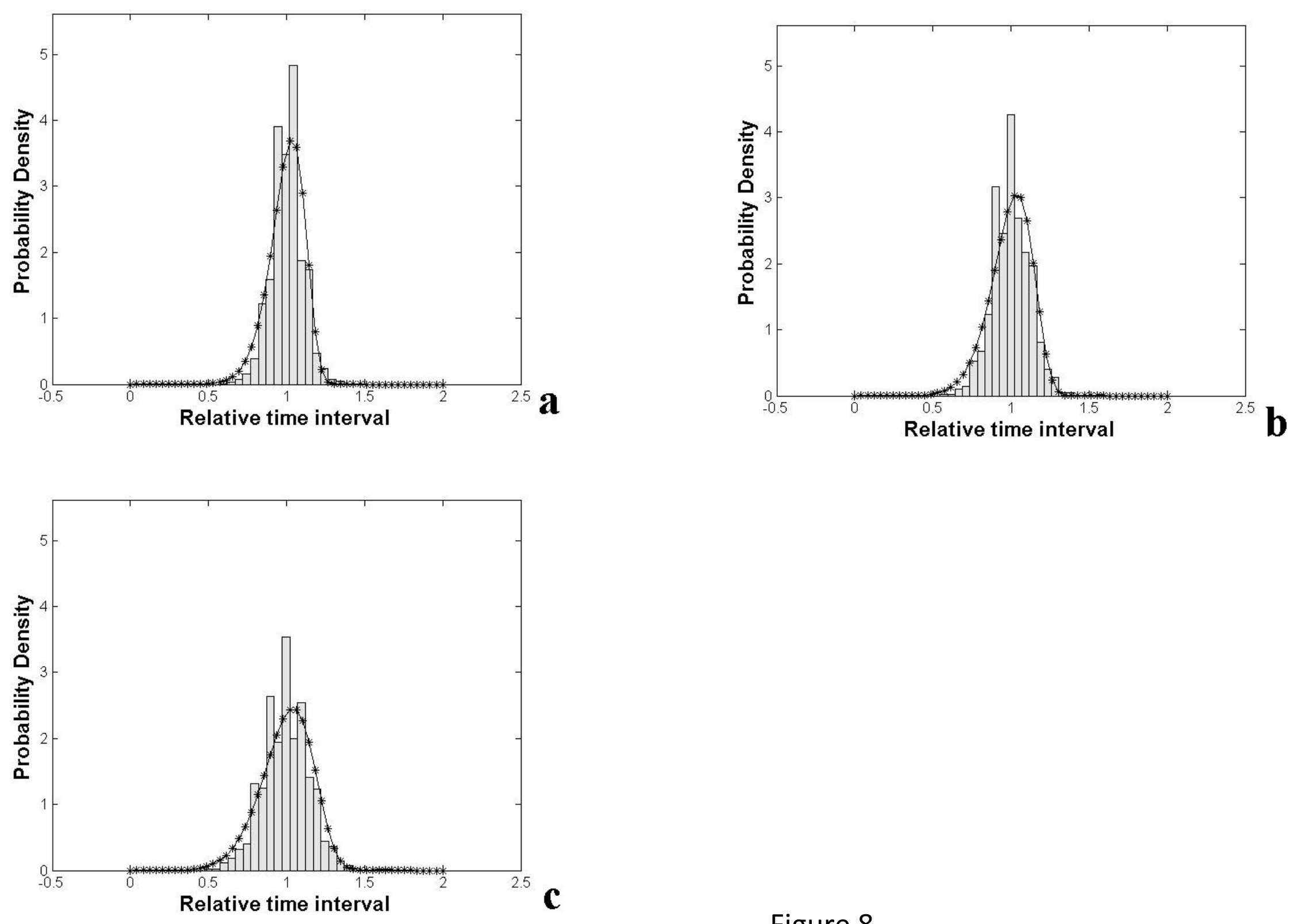

Figure 8 
Table 1. Minimal and maximal values of nucleation barriers for $T=823 \mathrm{~K}$

\begin{tabular}{|c|c|c|c|c|}
\hline $\begin{array}{c}\text { Disilane } \\
\text { pressure, Torr }\end{array}$ & $\begin{array}{c}\text { Average } \\
\text { concentration }\end{array}$ & $\begin{array}{c}\text { Average } \\
\text { supersaturation }\end{array}$ & $\frac{\Delta G_{\min }}{k T}$ & $\frac{\Delta G_{\max }}{k T}$ \\
\hline $4 \cdot 10^{-7}$ & 0,3053 & 0,04591 & 27,762 & 36.278 \\
\hline $5 \cdot 10^{-6}$ & 0,3088 & 0,04944 & 25,255 & 36,274 \\
\hline $3 \cdot 10^{-5}$ & 0,3118 & 0,05244 & 23,573 & 35,319 \\
\hline
\end{tabular}

Table 2. Dependence of different time characteristics on the disilane pressure $(T=823 \mathrm{~K})$.

\begin{tabular}{|c|c|c|c|c|c|}
\hline $\begin{array}{c}\text { Disilane } \\
\text { pressure, } \\
\text { Torr }\end{array}$ & $\begin{array}{c}\text { Average } \\
\text { waiting } \\
\text { time, } \mathrm{s}\end{array}$ & $\begin{array}{c}\text { Average } \\
\text { squared } \\
\text { deviation, s }\end{array}$ & $\begin{array}{c}\text { Dimensionless } \\
\text { standard } \\
\text { deviation }\end{array}$ & $\begin{array}{c}\text { Absolute } \\
\text { time } \\
\text { correlation, s }\end{array}$ & $\begin{array}{c}\text { Dimensionless } \\
\text { time } \\
\text { correlation }\end{array}$ \\
\hline $4 \cdot 10^{-7}$ & 12,456 & 1,238 & 0,099 & $-0,759$ & $-0,613$ \\
\hline $5 \cdot 10^{-6}$ & 0,996 & 0,118 & 0,119 & $-0,007$ & $-0,057$ \\
\hline $3 \cdot 10^{-5}$ & 0,166 & 0,025 & 0,149 & $-3,2 \cdot 10^{-4}$ & $-0,013$ \\
\hline
\end{tabular}

